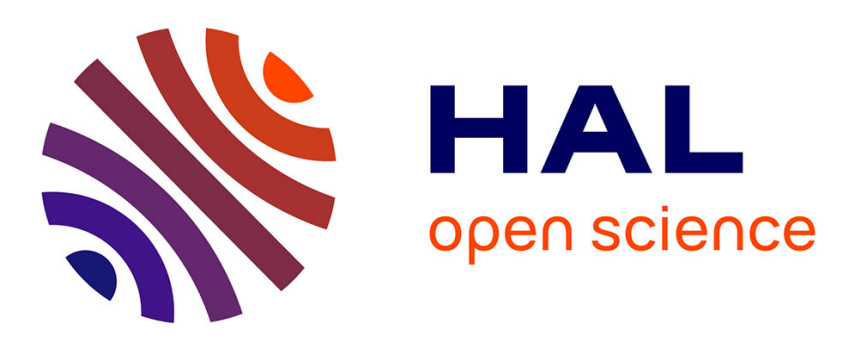

\title{
Shape tracing app for movement disorder detection
}

\author{
Vered Aharonson, Sarah Ward, David Anderson, David Milton Rubin, Michiel
}

Postema

\section{To cite this version:}

Vered Aharonson, Sarah Ward, David Anderson, David Milton Rubin, Michiel Postema. Shape tracing app for movement disorder detection. 2020 International SAUPEC/RobMech/PRASA Conference, Jan 2020, Cape Town, South Africa. pp.1-4, 10.1109/SAUPEC/RobMech/PRASA48453.2020.9041051 . hal-03192420

\section{HAL Id: hal-03192420 \\ https://hal.science/hal-03192420}

Submitted on 12 Apr 2021

HAL is a multi-disciplinary open access archive for the deposit and dissemination of scientific research documents, whether they are published or not. The documents may come from teaching and research institutions in France or abroad, or from public or private research centers.
L'archive ouverte pluridisciplinaire HAL, est destinée au dépôt et à la diffusion de documents scientifiques de niveau recherche, publiés ou non, émanant des établissements d'enseignement et de recherche français ou étrangers, des laboratoires publics ou privés. 


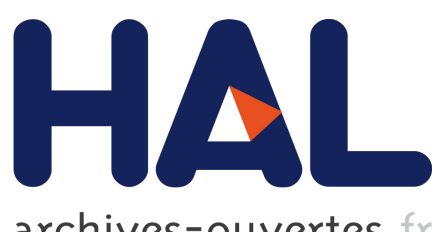

archives-ouvertes

\title{
Shape tracing app for movement disorder detection
}

\author{
Vered Aharonson, Sarah Ward, David Anderson, David Rubin, Michiel
}

Postema

\section{To cite this version:}

Vered Aharonson, Sarah Ward, David Anderson, David Rubin, Michiel Postema. Shape tracing app for movement disorder detection. 2020 International SAUPEC/RobMech/PRASA Conference, Jan 2020, Cape Town, South Africa. pp.1-4, 10.1109/SAUPEC/RobMech/PRASA48453.2020.9041051. hal-03192420

\section{HAL Id: hal-03192420 \\ https://hal.archives-ouvertes.fr/hal-03192420}

Submitted on 12 Apr 2021

HAL is a multi-disciplinary open access archive for the deposit and dissemination of scientific research documents, whether they are published or not. The documents may come from teaching and research institutions in France or abroad, or from public or private research centers.
L'archive ouverte pluridisciplinaire HAL, est destinée au dépôt et à la diffusion de documents scientifiques de niveau recherche, publiés ou non, émanant des établissements d'enseignement et de recherche français ou étrangers, des laboratoires publics ou privés. 


\section{Shape tracing app for movement disorder detection}

\author{
Vered Aharonson \\ School of Electrical and Information Eng. \\ University of the Witwatersrand \\ Johannesburg, South Africa \\ vered.aharaonson@wits.ac.za
}

\author{
David M. Rubin \\ School of Electrical and Information Eng. \\ University of the Witwatersrand \\ Johannesburg, South Africa \\ david.rubin@wits.ac.za
}

\author{
Sarah Ward \\ School of Electrical and Information Eng. \\ University of the Witwatersrand \\ Johannesburg, South Africa \\ sarah.ward@wits.ac.za
}

\author{
David Anderson \\ Donald Gordon Medical Centre \\ University of the Witwatersrand \\ Johannesburg, South Africa \\ david.anderson@wits.ac.za
}

\author{
Michiel Postema \\ School of Electrical and Information Eng. \\ University of the Witwatersrand \\ Johannesburg, South Africa \\ michiel.postema@wits.ac.za
}

\begin{abstract}
Shape tracing tests for the detection and assessment of hand movement disorders are predominantly performed manually in the presence of a clinician. These procedures are therefore labour intensive, expensive, and subjective. Digital tests have been proposed to automate this assessment process, to answer the need of affordable healthcare for all. A straightforward automation solution is a conversion of the shape tracing tests from pen and paper to a mobile device. This study implemements realtime dynamic touch detection on a mid-range tablet for shape tracing. The tracing app developed was tested on 20 movement disorder patients and 10 control subjects. The results convey that the interface allows for successful self-administration of the tests. For all subjects, the accuracy was successfully preserved in the real-time dynamic acquisition of the tracing process.

Index Terms-Tremor detection; shape tracing interface; realtime dynamic trace acquisition.
\end{abstract}

\section{INTRODUCTION}

Dysfunctions in hand movements, such as tremor and rigidity, severely hamper a person's quality of life. These dysfunctions are often the first symptoms of neurological movement disorders, the most common of which are Parkinson's disease, Essential Tremor and Huntington's disease [1]-[3]. A common screening and assessment of movement disorders is the shape drawing test, which provides visual indications of the symptoms involved. The subject has to trace the line of a predefined object with a pen on paper whilst a clinician observes the subject doing so. These assessments are predominantly performed in medical facilities, as they necessitate the physical presence of a clinician [4]. As a result, the administration of these tests is costly, limited the accessibility to all, and their interpretation is inherently qualitative and subjective [5].

The need for affordable healthcare and the fast evolution of digital technologies led to automation of many medical procedures. The use of digital data acquisition allows for a calculation of velocity and acceleration signals and an extraction of additional time and frequency-related features that enhance the characterisation of the hand movement disorder. Digital solutions were explored for quantitative and objective monitoring and diagnosis of hand movement disorders in the past two decades [6], [7]. These technologies and application greatly vary in terms of usability, complexity, efficacy, and accuracy. Electronic biosensor pens, wearable sensors on hand and fingers, and touch-screen devices have been reviewed [8]. In particular, solutions employing general purpose devices such as laptops, tablets and smartphones, have been considered [9], [10]. The apps developed for these media basically convert the pen-and-paper shape drawing into fingertip or stylus drawing. The digitised tests may require specialised and costly equipment. Tablets and smartphone screens differ in both display and data acquisition properties. Their accuracy may differ and hinder standardisation of the assessment. If a single, specific device is obligatory, the issues of affordability and accessibility remain, which is undesirable. Another weakness of proposed tablet apps is their usability: Uncomfortable and complex interfaces are unsuitable for self-administration to motor-impaired, sometimes elderly, users, which necessitates the presence of a healthcare professional to instruct and watch the user throughout the assessment [11]. The interpretation of the results may similarly be flawed: The dynamic digital data acquisition, compared to the static paper data has the potential of incorporating time information, which enables to quantify and interpret movement speed and acceleration. This information should be properly interpreted to avoid bias due to, e.g., natural slowness, age and computer skills. Both the acquisition and analysis of time properties of these apps may therefore be inaccurate. These properties may partially explain the limited usage and minimal acceptance of these solutions in hand movement disorders assessment.

This study proposes a new app to measure and characterise the severity of hand movement disorders. The aim of the design is to provide affordable assessment, to add real time accurate information, and to increase interface usability. A preliminary experiment on the three most common movement disorders: Parkinson's disease, Essential Tremor and Huntington's disease is performed. Data analysis and diagnostic accuracy performance, however, are not a focus of the paper. 


\section{Methods}

The application was developed on a ten-inch Lenovo Yoga 2 tablet, running the operating system Android (Google Inc., Mountain View, CA, USA), with 2 GB RAM, $1920 \times 1200$ pixels resolution and a built-in kick stand. This mid-range tablet provided a trade-off between cost-effectiveness versus sufficient display properties and processing power. A $2 \mathrm{~GB}$ RAM is generally sufficient for animation required for user interfaces of games and applications without any noticeable delay. The resolution of this tablet allows for improved accuracy when detecting the position of the user's finger while tracing the dot.

The software performs three core functions: user interface graphics, including animation of a reference dot along different shapes, detection of the user's finger movement along the screen, and storage of the collected data. The application was programmed in the mobile application development framework Apache Cordova (The Apache Software Foundation, Wakefield, MA, USA) in the programming language JavaScript (Ecma International, Genève, Switzerland), to support crossplatform portability [12]. The application graphics included five tracing shapes: a pulse, a straight horizontal line, a ramp, a sine wave, and a spiral, shown in Figure 1a-e.

The animation of the moving dot was performed using HTML-5 (Web Hypertext Application Technology Working Group, https://whatwg.org). This method allowed movements to be triggered at regular intervals and produced a smooth movement along the screen. Five speed options were provided in a setting page, so that the animation speed could be adjusted to a user's motor capabilities and age.

The animation dot moved along curved or straight line segments in a constant pace. The spiral shape presented a challenge since a constant speed animation is required in the animation motion for all shapes and segments. The dot movement was therefore calculated by integration between each pair of frames. A reduction of the computational complexity involved in integration is needed in this type of application, to afford as accurate a display as possible, since excessive computation slows down the animation and thus affects the speed. An approximation was made by making each incremental step a percentage larger than the previous step. The percentage was set using users' perception trials, incrementing the percentage and logging users' perception when observing the dot movement from the middle of the spiral outwards. The subjects were asked to evaluate for each chosen increment run, whether the speed appears constant or varying. The choice revealed that constant speed motion was observed by the human eye although in fact it increased slightly over time.

The detection of touch presented the greatest challenge in ensuring the reliability and consistency of the measurements. The captured data points are time stamped and should correspond to the animated reference dot timestamps. Touch detection functions are events that trigger a call-back whenever a motion on the screen is detected, which then allows the capture of positional data. The call-back was prone to delays due to the computer resource allocation. An examination of this property was performed using two software implementations: the built-in Cordova function onTouch, and a native Android function. Both implementations yielded that the triggering of the call-backs occurred only when there was sufficient processing power available. Therefore, the intervals between the captured touch events were irregular. Comparing the results of the two software and assessing the properties of the events logs confirmed that the limiting factors is the hardware limitations, prominently the screen refresh rate and the amount of accessible RAM. The timing reliability problem needed, therefore, to be resolved in the post processing stage. This data processing stage included interpolation of the reference data points, which occur at regular intervals, to form a continuous step-wise smooth function, and a resampling of the acquired data points to provide time-alignment with this reference data.

Another pitfall that may hamper the accurate data acquisition needed for such applications is the data completeness and accuracy, which pertains to database storage issues. The acquired movement data produced of 5MB. A simple database used in low-cost applications may produce occasional data corruption errors when writing such large files. To prevent any loss of data due to this problem a backup of the data is stored temporarily in the device's local storage. Data check needs to be performed to ensure that the data are correctly stored in the database, before the device local storage can be cleared. A core part of the design of the application is the user interface. This is an important aspect in any application used by people, but as the prospective users of the current application may suffer from motor disorders and may be elderly who are not be comfortable with technology, usability is a crucial issue. A second user of the system is a healthcare professional who may use the application as a diagnostic aid. The interface for this type of user should be very simple and clear, to enable usage in a busy clinic setting.

The clinician interface consists of maximum of 2 screens. The initial interface displays 3 buttons with the options: log-in of an existing patient or add a new patient. A second screen correspondingly displays a search for an existing patient or a form for new user registration. Both options end in a setting button to adjust the moving dot speed.

The patient interface was designed to be as intuitive as possible and required minimal reading of instructions. Figure if shows a shape tracing screenshot: The reference dot moved to guide the subjects in the shape tracing, and the instructions explained that they should attempt to keep their fingertip on this dot at any given time. Crosshairs added to the dot enabled the subjects to deduce its centre when their fingertip covered the dot. The green and red blocks indicated the start and end of the required tracing. The reference dot started moving only when a finger touch was detected in the green area. The system moved to the next shape only after the tracing has reached the red region. A restart button and option was provided to reset each test. A shape tracing test could not be stopped midway and a subject could not go back to previous shape 
tests. This was to ensure that all tests were completed in a short single sitting and that the data collected were consistent across subjects.

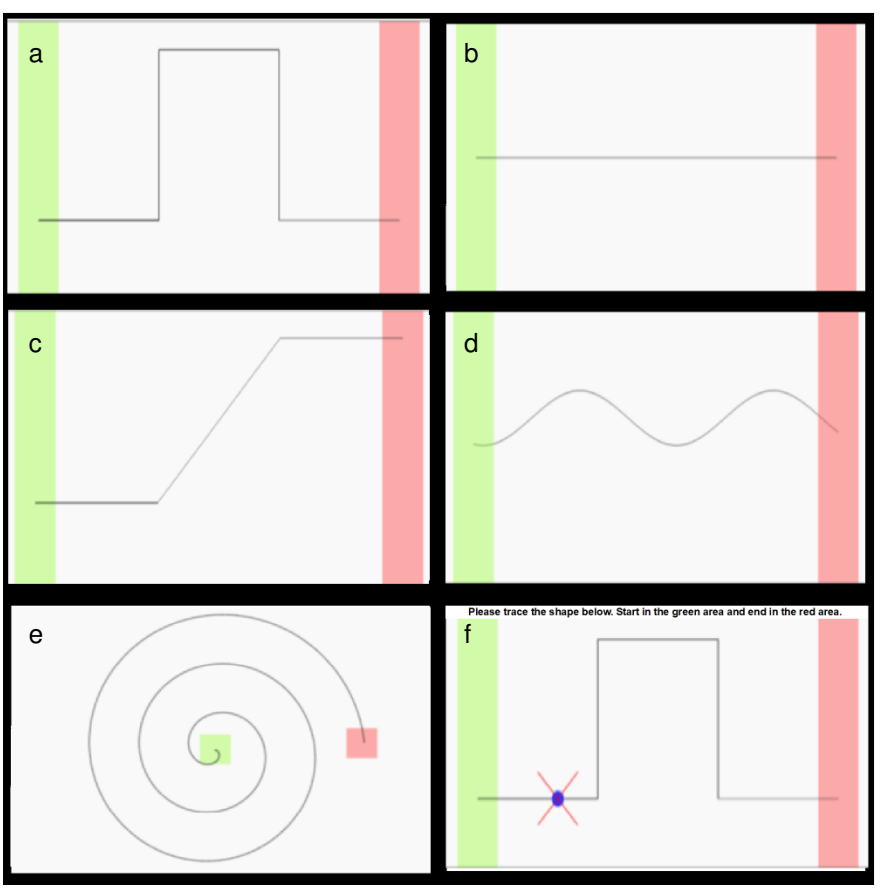

Fig. 1. Tracing shapes used in the application: pulse (a), straight line (b), ramp (c), sine wave (d), spiral (e). The tracing follows a moving dot, starting in the green rectangle and ending in the red rectangle (f).

The built-in kickstand of the tablet provided comfortable screen angle and position to the subjects and was reliably reproducible in repeating trials. The height of the table the tablet was placed on and its distance from the subject needed to be adjusted for each subject's comfort.

Twenty patients with movement disorders and ten agematched control participants were recruited to test the app. Nine Parkinson's disease patients, six Essential Tremor patients and five Huntington's disease patients. The age range was from 34 to 85 . Informed consent was obtained from all subjects and their data were anonymised as per the approved ethical clearance requirements. Following an experimenter input in the clinician's user interface screens, the users were instructed by the application and traced the shapes using their index finger, trying to keep up with the dot that moves along the shape. A maximal speed for the dot motion was set for all users, to examine the usability at highest difficulty level.

Another measure to ensure data collection reliability, the first shape, a pulse, was repeated at the end of the test. This enabled data normalisation, which is necessary as users typically get more accustomed to the system the longer they use it. The use of the same graph for the first and last test allows this factor to be taken into consideration.

The focus of this preliminary study was not the diagnostic capability of the app, but rather on its usability features. The performance measures were as follows: Data completeness was assessed based on the amount of data lost, in percentage.
Statistical analysis of the touch sampling intervals before and after the data alignment compensation was performed.

\section{RESULTS}

All patients and control subjects were able to complete all the tests and none reported having difficulty. The full experiment session took under 15 minutes for each of the subjects. Three patients needed to restart a shape tracing test once or twice. No subject required more than three trials of a test.

No data were lost throughout any of the tests.

The touch detection data yielded that the range of time intervals between captured samples in the acquired data before correction was 0.17 seconds, with an average of 0.024 seconds and a standard deviation of 0.013 seconds. Post processing was successful in mitigating this issue and aligning the data and reference. An example of the corrected touch data and its reference is shown in Figure 2a. The graph depicts the $\mathrm{x}$ axis of the spatial position of a pulse tracing as a function of time. The reference dot position in red and the user's tracing in blue. The two graphs do not overlap exactly due to a delay introduced by the patient's response time and not due to misalignment of the data. This delay was reduced as subjects proceeded in the tests, which imply that they got used to the speed of the moving dot and manage to track it more closely. The last shape tracing of an identical pulse confirmed this assumption. Figure 2a displays a performance of a control subject, whereas Figure 2b-d display respective examples of tracing tests of a pulse signal of a Parkinson's disease patient, an Essential Tremor patient and a Huntington's disease patient.
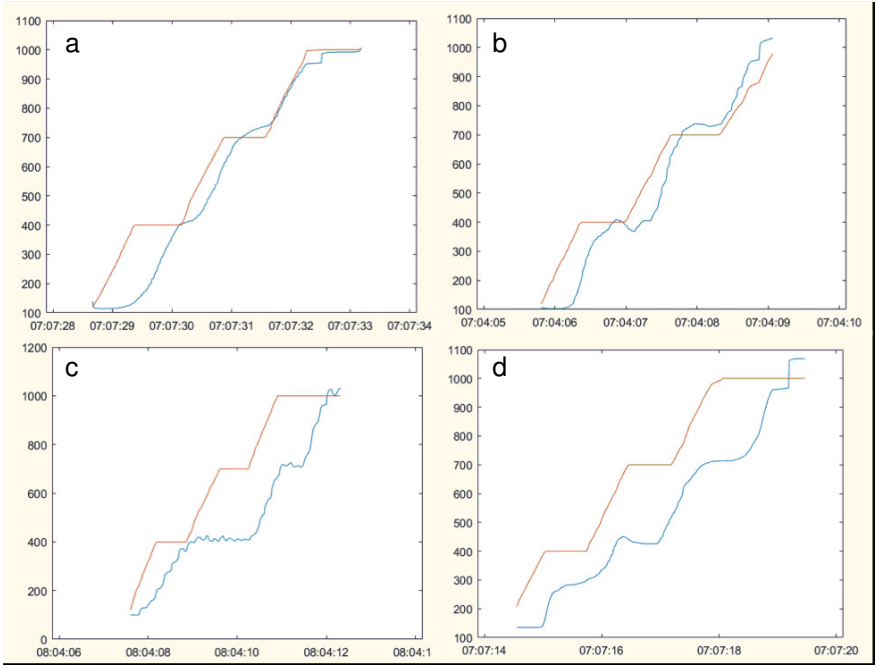

Fig. 2. Time-aligned data. The subject data are represented in blue and the reference dot trajectory in red. The horizontal axis is the absolute time of measurement and the vertical axis is the $x$-coordinate of the path drawn in pixels. Examples from a control subject (a), a Parkinson's patient (b), an Essential Tremor patient (c), and a Huntington's patient (d). 


\section{DISCUSSION AND CONCLUSIONS}

The conversion of pen-and-paper shape-drawing assessment to a tablet app is an intricate process. Affordability, usability and accuracy related pitfalls need to be carefully studied and avoided. In this study we addressed some of these pitfalls in the design of a new tablet app and studied this design on twenty movement disorders patients and ten controls. All users were able to complete the assessment introduced by the app, without assistance from the experimenters and healthcare professions. This result corroborates previous studies performed on patients with cognitive impairments, where a patient-centred design made the assessment software usable for these patients and elderly controls [13], [14]. The increased interface usability in the current study may make the full time presence of the clinician during testing superfluous.

The real-time control feature of forcing the shape tracing pace by the moving dot could enhance accuracy by reducing bias of natural, non-movement disorder-related differences between subjects. A thorough examination was performed on the irregularity of the timing accuracy of touch screen events. These timing inaccuracies may severely hamper data reliability. The issues that stemmed from hardware limitations could be present in any tablet and need to be both assessed and corrected. Our examination demonstrated that these hardware issues cannot be resolved by the event acquisition software and were corrected in a post-acquisition stage. The dynamic trait of the app' interface brought to light another issue in the implementation of constant control pace to the guiding animation. The issue was specific to the spiral shape, which is one of the most popular shape in the traditional pen and paper as well as in their computerised versions. The proposed solution implemented a low-complexity adaptation to offer this constant pace capability.

This study was conducted using a mid-range tablet. The fast progress in tablets may be able to implement today's midrange tablet properties into low-range device in a year or two, which will make these devices accessible to an even wider audience. cite This paper focusses on the assessment app's data acquisition. The accuracy issues will be further explored in a comprehensive analysis study of the acquired data, to examine the effect of the enhanced accuracy on the classification of movement disorders and their severity. This future study will contain a larger cohort of patients from the different types of movement disorders diseases and will collect a comprehensive clinical data on the patients.

The components of the app are similar to most tablet apps: user interface, data acquisition and storage. Since this special app is used in medical settings and the data were to be used for medical purposes, considerations that are sometimes overlooked need to be examined. A better quality in user interaction and data storage could benefit other, non-medical apps as well.

\section{REFERENCES}

[1] M.A. Smith, J. Brandt, and R. Shadmehr, "Motor disorder in Huntington's disease begins as a dysfunction in error feedback control," Nature, vol. 403 , p. 544, 2000.

[2] G. Deuschl, R. Wenzelburger, K. Löffler, J. Raethjen, and H. Stolze, "Essential tremor and cerebellar dysfunction clinical and kinematic analysis of intention tremor," Brain, vol. 123, pp. 1568-1580, 2000.

[3] G. Abbruzzese and A. Berardelli, "Sensorimotor integration in movement disorders," Mov. Disord., vol. 18, pp. 231-240, 2003.

[4] J. Jankovic, "Parkinson's disease: clinical features and diagnosis," J. Neurol. Neurosurg. Psychiatry, vol. 79, pp. 368-376, 2008.

[5] M. A. L. Lopes, H. P. Ferreira, J. C. Carvalho, L. Cardoso, and C. André, "Screening tests are not enough to detect hemineglect," Arq. Neuro-Psquiat., vol. 65, pp. 1192-1195, 2007.

[6] L. Collins, G. Cummins, and R. A. Barker, "Parkinson's disease: diagnosis and current management," Prescriber, vol. 26, pp. 16-23, 2015.

[7] P. H. Kraus and A. Hoffmann, "Spiralometry: computerized assessment of tremor amplitude on the basis of spiral drawing," Mov. Disord., vol. 25, pp. 2164-2170, 2007.

[8] D. Impedovo and G. Pirlo, "Dynamic handwriting analysis for the assessment of neurodegenerative diseases: a pattern recognition perspective," IEEE Rev. Biomed. Eng., vol. 12, pp. 209-220, 2019.

[9] J. Westin, S. Ghiamati, M. Memedi, D. Nyholm, A. Johansson, M. Dougherty, and T. Groth, "A new computer method for assessing drawing impairment in Parkinson's disease," J. Neurosci. Meth., vol. 190, pp. 143-148, 2010.

[10] M. Isenkul, B. Sakar, and O. Kursun, "Improved spiral test using digitized graphics tablet for monitoring Parkinson's disease," in: Proceedings of the International Conference on e-Health and Telemedicine, 2014, pp. $171-175$.

[11] V. Aharonson and A. D. Korczyn, "Computerized Methods for Cognitive Testing," in: Eldercare Technology for Clinical Practitioners, M. Alwan, R. A. Felder, Eds., Berlin: Springer, 2008, pp. 203-215.

[12] S. Bosnic, I. Papp, and S. Novak, "The development of hybrid mobile applications with Apache Cordova," in: Proceedings of the 24th Telecommunications Forum, 2016, pp. 1-4.

[13] V. Aharonson and A. D. Korczyn, "Human-computer interaction in the administration and analysis of neuropsychological tests," Comput. Meth. Prog. Bio., vol. 73, pp. 43-53, 2004.

[14] V. Aharonson, I. Halperin, and A. D. Korczyn, "Computerized diagnosis of mild cognitive impairment," Alzheimers Dement., vol. 3, pp. 23-27, 2007. 